\title{
Open
}

\section{Innate immune activation in neonatal tracheal aspirates suggests endotoxin-driven inflammation}

\author{
Katheryn E. Nathe ${ }^{1-4}$, Christy J. Mancuso ${ }^{1}$, Richard Parad ${ }^{1,2,4}$, Linda J.Van Marter ${ }^{1,2,4}$, Camilia R. Martin ${ }^{4,5}$, Liat Stoler-Barak', \\ Victoria J. Philbin ${ }^{1,4}$, Michele F. Phillips ${ }^{2}$, Christine D. Palmer ${ }^{1,4}$ and Ofer Levy ${ }^{1,4}$
}

BACKGROUND: Tracheal aspirates (TAs) from critically ill neonates accumulate bacterial endotoxin and demonstrate mobilization of endotoxin-binding proteins, but the potential bioactivity of endotoxin in TAs is unknown. We characterized innate immune activation in TAs of mechanically ventilated neonates.

METHODS: Innate immune activation in TAs of mechanically ventilated neonates was characterized using a targeted 84-gene quantitative real-time (qRT) PCR array. Protein expression of cytokines was confirmed by multiplex assay. Expression and localization of the endotoxin-inducible antimicrobial protein Calgranulin C (S100A12) was assessed by flow cytometry. Endotoxin levels were measured in TA supernatants using the Limulus amoebocyte lysate assay.

RESULTS: Analyses by qRT-PCR demonstrated expression of pattern recognition receptors, Toll-like receptor-nuclear factor $\mathrm{KB}$ and inflammasome pathways, cytokines/chemokines and their receptors, and anti-infective proteins in TA cells. Endotoxin positivity increased with postnatal age. As compared with endotoxin-negative TAs, endotoxin-positive TAs demonstrated significantly greater tumor necrosis factor (TNF), interleukin (IL)-6, IL-10, and serpin peptidase inhibitor, clade E, member 1 (SERPINE1) mRNA, and IL-10, TNF, and IL-1 $\beta$ protein. Expression of S100A12 protein was localized to TA neutrophils.

CONCLUSION: Correlation of endotoxin with TA inflammatory responses suggests endotoxin bioactivity and the possibility that endotoxin antagonists could mitigate pulmonary inflammation and its sequelae in this vulnerable population.

P ulmonary disease is a major cause of morbidity in premature infants (1). Several lines of evidence suggest that innate immune activation may play important roles in the development of respiratory diseases early in life (2). Preterm, mechanically ventilated neonates are predisposed to bacterial tracheal colonization, which is associated with cytokine responses that may contribute to pulmonary inflammation (3). Indeed, microbial colonization and production of cytokines and chemokines such as interleukin (IL)-1 $\beta$ and CXCL8, particularly early in the course of intubation, has been associated with subsequent respiratory disease $(4,5)$.
Innate immune activation in the newborn is incompletely characterized (6), especially with respect to the respiratory tract (7). Pathogen-associated molecular patterns are detected by pattern recognition receptors, including Toll-like receptors (TLRs) and the intracellular inflammasome complex, pathways that induce acute inflammatory responses $(8,9)$. TLRs signal via adaptor molecules such as MyD88 (10), and downstream serinethreonine kinases to induce cytokines via activation of transcription factors including nuclear factor $\mathrm{\kappa B}(\mathrm{NF \kappa B})$ and interferon regulatory transcription factor family members $(11,12)$.

Bacterial endotoxin found in all Gram-negative bacteria is one of the most potent known activators of the TLR pathway; it is active at picogram concentrations. Detection of bacterial endotoxin by the endotoxin receptor complex composed of CD14/TLR4/MD2 induces production of cytokines, including tumor necrosis factor (TNF) and IL-6 $(11,13,14)$, IL-1 family members via the inflammasome (9), antiinflammatory cytokines such as IL-10 (15), and chemokines that attract infiltrating polymorphonuclear leukocytes (PMNs) and monocytes to sites of infection $(16,17)$.

Cells respond to secreted cytokines and chemokines via cognate receptors (18), leading to further production of inflammatory response genes, including complement proteins and anti-infective proteins and peptides (19). These are secreted into the respiratory tract in response to infection via direct synthesis by tracheobronchial epithelial cells (20) and/or by cytokine/ chemokine-based recruitment and activation of PMNs (21). Regulatory molecules such as heme-oxygenase-1 (HMOX1) and serpin peptidase inhibitors can further modulate host responses $(22,23)$. Overall, little is known regarding the relative expression of these pathways in the airways of intubated preterm newborns.

We have previously reported the presence of Gram-negative bacterial endotoxin in tracheal aspirates (TAs), along with mobilization of endotoxin-directed proteins such as sCD14, lipopolysaccharide binding protein and bactericidal/permeability increasing protein (24). This study raised the possibility that endotoxin may contribute to respiratory inflammation in this setting. However, neither the scope of innate immune activation in TAs nor the potential correlation of endotoxin with inflammatory responses has been characterized.

\footnotetext{
'Department of Medicine, Children's Hospital Boston, Boston, Massachusetts; ${ }^{2}$ Department of Newborn Medicine, Brigham and Women's Hospital, Boston, Massachusetts; ${ }^{3}$ Department of Pediatrics, Massachusetts General Hospital for Children at North Shore Medical Center, Salem, Massachusetts; ${ }^{4} \mathrm{Harvard}$ Medical School, Boston, Massachusetts; ${ }^{5}$ Department of Neonatology Beth Israel Deaconess Medical Center, Boston, Massachusetts. Correspondence: Ofer Levy (ofer.levy@childrens.harvard.edu) 
Table 1. Subject characteristics

\begin{tabular}{lc} 
Subject characteristic & \\
Subjects $(n)$ & 53 \\
TA samples collected, total $(n)$ & 69 \\
Male \% & $62 \%$ \\
Gestational age (wk) & $27.8 \pm 4.2$ \\
Gestational age, range (wk) & $23-39$ \\
Postnatal age, all samples (d) & $16.5 \pm 16.2$ \\
Postnatal age, range, all samples (d) & $0-71$ \\
Birth weight (g) & $1,137 \pm 842$ \\
Endotoxin-positive \% & $40 \%$ \\
Bronchopulmonary dysplasia \% & $55 \%$ \\
\hline Values are represented as mean \pm SD unless otherwise specified
\end{tabular}

Values are represented as mean $\pm S D$, unless otherwise specified.
To characterize innate immune activation in neonatal TAs in relation to endotoxin, we employed a targeted transcriptional profiling approach using quantitative realtime (qRT)-PCR using TA samples of limited volume and cell number. Our objectives were to (i) determine the feasibility of the qRT-PCR approach to TA transcriptional profiling, (ii) validate this approach by characterizing expression of select proteins, and (iii) assess potential correlations of innate immune expression with the presence of endotoxin.

Herein, we demonstrate the feasibility and validity of a qRTPCR approach to characterize activation of innate immune pathways in neonatal TAs, revealing broad transcriptional activation

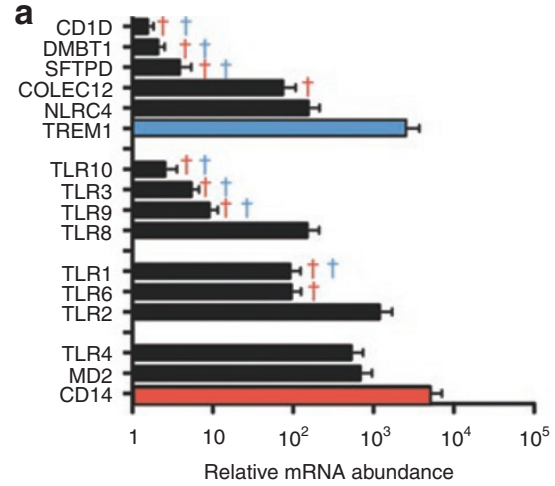

b

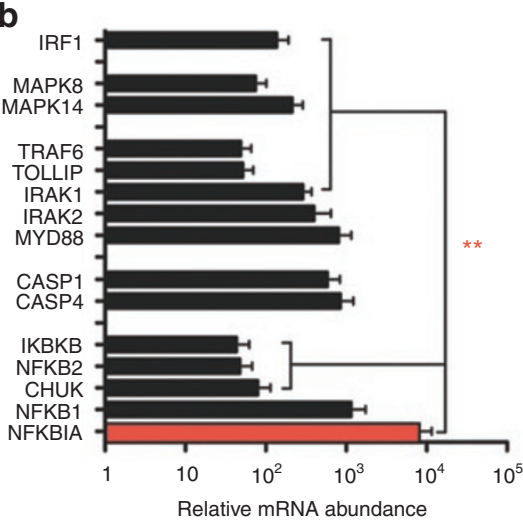

C

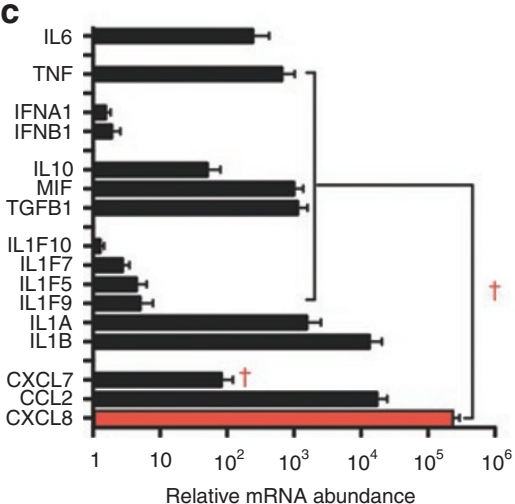

d

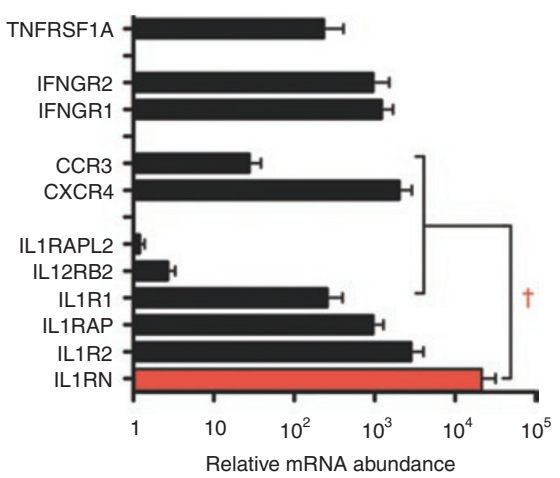

e

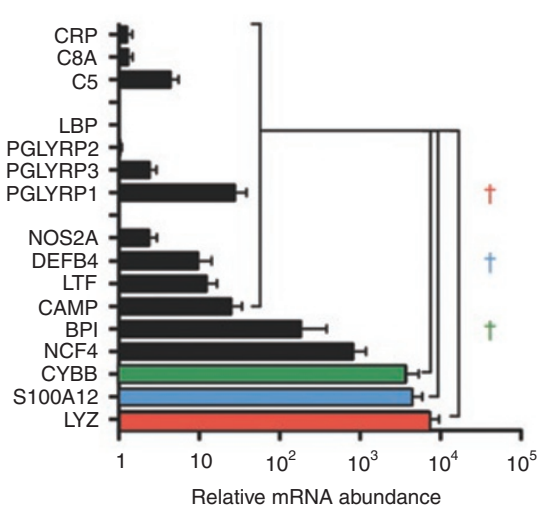

f

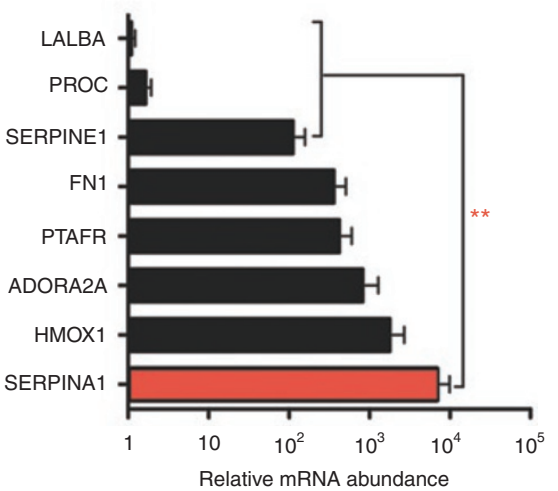

Figure 1. Innate immune transcriptome of TA pellets. TA pellet mRNA ( $n=25$, except for CXCL8, for which $n=14)$ was analyzed by quantitative real-time PCR. mRNA levels of 82 genes are shown as mean \pm SEM mRNA abundance and grouped by function as follows: (a) pattern recognition receptors, (b) intracellular signaling, (c) cytokine/chemokines, (d) cytokine/chemokine receptors, (e) anti-infective, and (f) other. All mRNA transcripts except for LBP were consistently detected in all subjects. Statistical analyses by one-way ANOVA, Bonferroni posttest, ${ }^{* *} P<0.01,{ }^{\dagger} P<0.001$. Colored stars and daggers signify statistically significant differences from the correspondingly colored bar within each graph. LBP, lipopolysaccharide binding protein; TA, tracheal aspirate. 

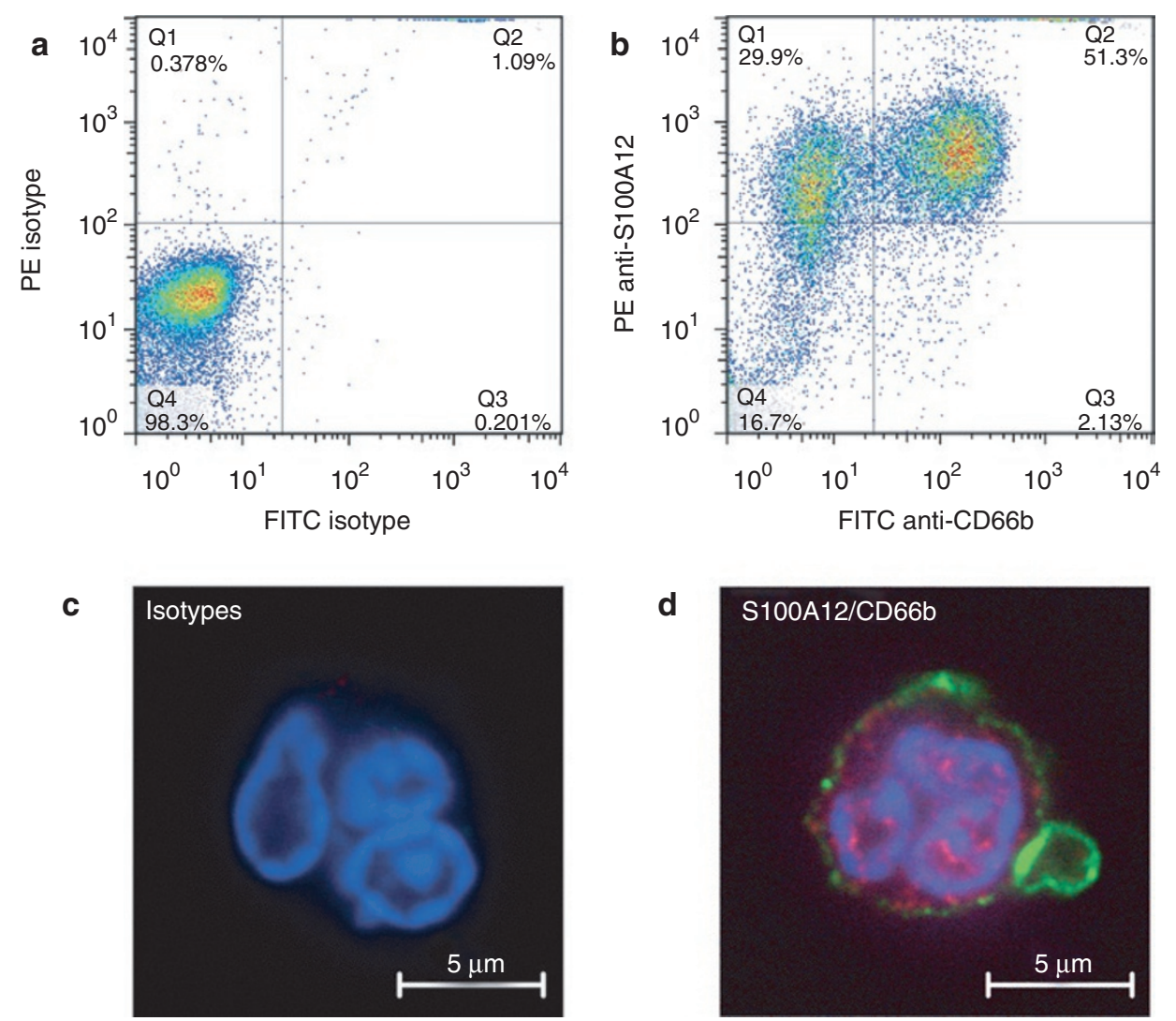

Figure 2. S100A12 protein expression localizes to TA PMNs. Representative dot plot of TA cells stained with (a) fluorescein isothiocyanate (FITC) and R-phycoerythrin (PE) isotype control antibodies and (b) antibodies for cell-surface antigens FITC-CD66b (PMN) and intracellular PE-S100A12 by flow cytometry. Representative confocal fluorescent microscopy (100X) of TA cells stained with (c) isotype control antibodies, or (d) antibodies against CD66b (green) and S100A12 (red), both with DRAQ5 nuclear stain (blue). (d) A characteristic CD66 b/S100A12 PMN. PMN, polymorphonuclear leukocyte; TA, tracheal aspirate.

of pattern recognition receptors, signaling molecules, anti-infective proteins, and cytokines. Detected gene expression varied by as much as $5 \log$ orders of magnitude. Expression of several transcripts was confirmed at the protein level, including multiple cytokines, as well as mobilization of the endotoxin-inducible anti-infective protein Calgranulin C (S100A12) localized to TA PMNs. Moreover, our studies have revealed that the presence of endotoxin in TA supernatants correlates with expression of inflammatory cytokines such as TNF and IL- $1 \beta$, suggesting that bioactive endotoxin could contribute to respiratory inflammation and its sequelae.

\section{RESULTS}

\section{Study Population}

Demographics and relevant clinical characteristics of the study subjects appear in Table 1. Samples from infants $(n=53)$ with gestational age (GA) range of $23-39 \mathrm{wk}$ are represented, with a postnatal age range of $0-71 \mathrm{~d}$.

\section{mRNA Transcript Yield and Abundance}

TA pellets, containing $1.97 \times 10^{5}-1.32 \times 10^{7}$ total viable cells, all yielded sufficient quantity $(56-1,280 \mathrm{ng})$ and quality mRNA for downstream qRT-PCR, allowing measurement of relative mRNA abundance of 86 genes in the innate immune pathway. RNA yield $(\mu \mathrm{g} / \mathrm{ml})$ positively correlated with cellularity of the TA pellets. All
mRNA transcripts except for lipopolysaccharide binding protein, which is predominantly an acute-phase liver product, were consistently detected in all subjects tested (25/25). Figure 1 shows mRNA levels as mean \pm SEM mRNA relative abundance grouped by function. CD14, a component of the endotoxin receptor, and TREM-1, an endotoxin-inducible receptor that amplifies lipopolysaccharide induced inflammation (25), demonstrated greater expression than multiple other pattern recognition receptors (Figure 1a). NFKBIA, a component of the NFKB signaling pathway, was most abundantly expressed within the intracellular signaling transcripts (Figure 1b). The IL-1 receptor antagonist gene $(I L-1 R N)$ had the highest expression of the cytokine/chemokine receptors (Figure 1c), with CXCL8, a neutrophil chemoattractant, being expressed at higher levels than most other cytokines/ chemokines (Figure 1d). Among the anti-infective proteins and peptides, lysozyme, cytochrome b245 $\beta$-chain, and S100A12 demonstrated the greatest expression (Figure 1e). In addition, serpin peptidase inhibitor clade A member 1 (SERPINA1) was most abundantly expressed within the "other" grouping (Figure 1f).

\section{Assessing S100A12 as a Novel TA Transcript}

As S100A12 had not been previously reported in human neonatal TAs, we characterized its expression at the protein level by enzyme-linked immunosorbent assay (ELISA). Levels of S100A12 positively correlated with TA cell counts as assessed 
by hemocytometry $(n=19$; Spearman $r=0.59, P<0.01)$. Flow cytometry of TA cells for surface CD66b (PMNs) and intracellular S100A12 demonstrated that $\mathrm{CD} 66 \mathrm{~b}^{+} / \mathrm{S} 100 \mathrm{~A} 12^{+} \mathrm{PMNs}$ represent $>50 \%$ of the total TA cell population (Figure 2a,b). This localization was confirmed by confocal microscopy demonstrating TA cells stained for CD66b (green), S100A12 (red), and nuclei (blue; Figure 2c,d).

\section{Characterization of TA Cytokine Protein Concentrations}

To further validate our mRNA array results, IL-10, TNF, IL-1 $\beta$, IL-6, CXCL8, and CCL2 protein concentrations were measured by multiplex assay. Cytokine/chemokine transcript levels correlated with respective protein levels (Figure 3a), suggesting that the qRT-PCR approach may be of value in predicting the presence of TA inflammatory markers. All six cytokines were detectable, with IL-10 produced at the lowest levels $(\sim 10 \mathrm{pg} /$ $\mathrm{ml})$, and CCL2 produced at the highest level $(\sim 10,000 \mathrm{pg} / \mathrm{ml}$; Figure $3 b)$. Cytokine concentrations did not significantly vary by GA, birth weight, sex, subsequent bronchopulmonary dysplasia diagnosis, or mean fraction of inspired oxygen $\left(\mathrm{FiO}_{2}\right)$ exposure (data not shown).

\section{Endotoxin-Positive TAs Demonstrate Increased mRNA Expression}

As the observed pattern of TA gene transcription and cytokine production suggested a possible response to endotoxin (26), we next measured endotoxin in TA supernatants by Limulus amoebocyte lysate assay $(<0.2$ endotoxin units $(\mathrm{EU}) / \mathrm{ml}, n=$ $32 ; 0.2-1 \mathrm{EU} / \mathrm{ml}, n=7 ;>1-100 \mathrm{EU} / \mathrm{ml}, n=5 ;>100 \mathrm{EU} / \mathrm{ml}, n=$ 9). Upon analysis of TAs for which bacterial culture was available, recovery of Gram-negative bacteria significantly correlated with higher endotoxin levels (data not shown). Of the 25 TA pellets assayed by qRT-PCR, 24 also had TA supernatants available for endotoxin testing via Limulus amoebocyte lysate assay. As compared with endotoxin-negative samples, endotoxin-positive TAs demonstrated significantly greater mRNA expression of IL-6, IL-10, TNF, and serpin peptidase inhibitor, clade E, member 1 (SERPINE1) (Figure 4a,b), suggesting endotoxin-induced activation of gene transcription.

\section{TA Supernatant Cytokines Correlate With Postnatal Age and Endotoxin Positivity}

We next assessed whether protein biomarkers correlated with the presence of endotoxin in TAs. Consistent with a lack of upregulation of the S100A12 mRNA transcript in endotoxinpositive TA pellets (Figure 4a), concentrations of S100A12 protein were not significantly higher in endotoxin-positive than in endotoxin-negative TA supernatants (data not shown). However, TA supernatants from intubated neonates at postnatal day of life $(\mathrm{DOL}) \geq 4 \mathrm{~d}$ (mean $\mathrm{DOL}=15.7 \mathrm{~d}$ ) expressed higher levels of TNF, IL-1 $\beta$, and CXCL8 than those at DOL $<4$ $\mathrm{d}$ (mean DOL $=1.2 \mathrm{~d}$; Figure 5a). Indeed, TNF, IL-1 $\beta$, and CXCL8 increased significantly from DOL $0-3$ to DOL $4-9$ (Figure 5b). Postnatal DOL also correlated with levels of endotoxin (Figure 5c), and endotoxin-positive TA supernatants contained significantly greater concentrations of IL-10,
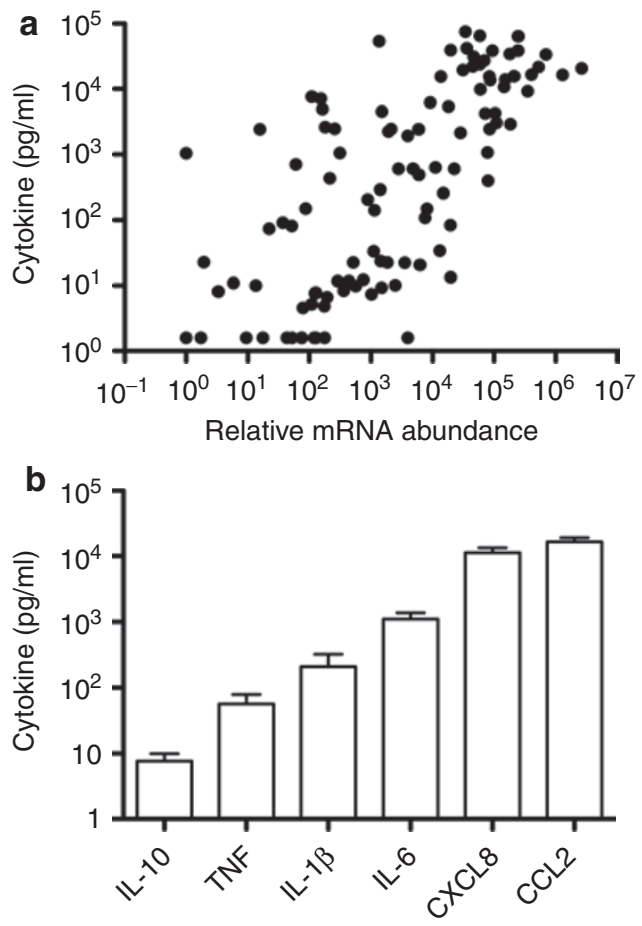

Figure 3. Correlation of TA cytokine expression at mRNA and protein levels. (a) Cytokine transcription levels correlated with respective TA supernatant cytokine protein levels measured using Milliplex Cytokine 6-Plex assay ( $n=43-46,115$ data points, Spearman $r=0.73, P<0.001)$; (b) IL-10, TNF, IL-1 $\beta, I L-6, C X C L 8$, and CCL2 were all present at the protein level. TA supernatant cytokine protein levels measured using Milliplex Cytokine 6-Plex assay are shown in $\mathrm{pg} / \mathrm{ml}(n=43-46)$. IL, interleukin; TA, tracheal aspirate; TNF, tumor necrosis factor.

TNF, and IL- $1 \beta$ protein than endotoxin-negative TA supernatants (Figure 5d), suggesting endotoxin-driven transcription of these cytokines. Accordingly, IL-10 levels increased significantly in endotoxin-positive TAs from DOL 0-3 to DOL 4-9, then decreased over time (Figure 5e).

\section{DISCUSSION}

We have characterized for the first time an innate immune transcriptome in the TAs of critically ill, mechanically ventilated human newborns. Although transcription of nearly all of the genes selected (84/85) was detectable, expression of innate immunity genes varied substantially across five orders of magnitude. Verification of expression at the protein level (cytokines/ chemokines, S100A12) indicated that mRNA expression was predictive of protein expression, and generally proportional in magnitude, indicating the robustness of this approach.

The most abundantly expressed anti-infective proteins/peptides in TAs were lysozyme, S100A12, and cytochrome b245 $\beta$-chain, a phagocyte oxidase component important for oxygen radical production. Expression of the PMN granule-associated lysozyme and bactericidal/permeability increasing protein in neonatal TAs have been previously reported (24). Of note, we are the first to demonstrate PMN-associated expression of S100A12 in neonatal TAs. In adult humans, S100A12 is predominantly expressed in PMNs (27) and in monocytes (28), 

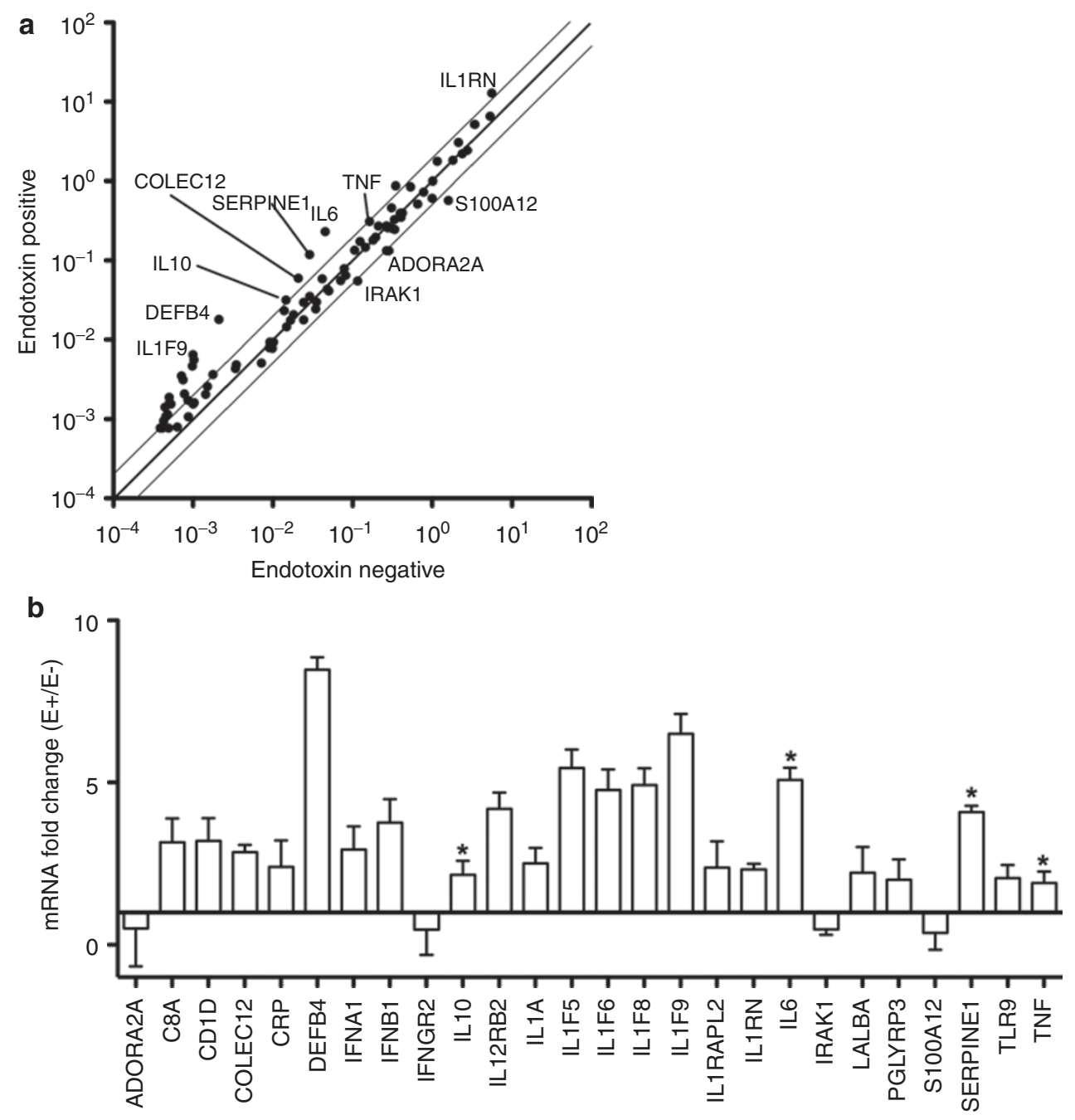

Figure 4. Endotoxin in TA supernatants correlates with transcription of innate immune genes. (a) Of 25 TA pellets assayed for mRNA transcripts, 24 had TA supernatants available for endotoxin testing via LAL assay. Endotoxin-positive TAs $(n=6)$ demonstrated greater mRNA expression of IL-6, IL-10, TNF, and SERPINE1 ( $P<0.05$, Student's $t$ test) as compared with endotoxin-negative samples $(n=18)$. (b) The data in (a) are plotted as a ratio of expression in endotoxin-positive vs. endotoxin-negative ( $E+/ E-)$ samples for transcripts with differences greater than twofold ( $P<0.05)$. IL, interleukin; $L A L$, Limulus amoebocyte lysate; SERPINE1, serpin peptidase inhibitor, clade E member 1;TA, tracheal aspirate; TNF, tumor necrosis factor.

and its expression can be enhanced in some settings by the introduction of endotoxin in vitro or into the bloodstream in vivo $(29,30)$. In contrast, in our study, S100A12 protein correlated with TA cell count, but neither S100A12 mRNA nor protein levels were higher in endotoxin-positive as compared with endotoxin-negative TA samples. We speculate that the mechanisms of S100A12 mobilization from neutrophils in the intubated respiratory tract of the neonate are distinct from those in adult studies of acute/single endotoxin exposure in vitro or in the peripheral blood compartment in vivo.

Upon mobilization to the respiratory tract, S100A12 may play important roles in host defense and inflammation. S100A12 is microbicidal through its C-terminal peptide, calcitermin, which kills Gram-negative bacteria and Candida albicans (31). S100A12 is also released from activated or damaged cells under stress (32) and can bind the receptor for advanced glycation end-products on pulmonary type I alveolar cells and macrophages, triggering $\mathrm{NF \kappa B}$ signaling and expression of pro-inflammatory cytokines and adhesion molecules (33). Accordingly, S100A12 is elevated in bronchoalveolar lavage fluid of adults with acute respiratory distress syndrome $(29,34)$ or chronic lung disease (34). Thus, S100A12 could help ward off infection and potentially contribute to inflammation, and may also serve as a biomarker in this setting.

With respect to cytokines and chemokines, we observed abundant mRNA expression of CXCL8, CCL2, and IL-1 $\beta$ $\left(>10^{3}\right.$ relative mRNA abundance). These were confirmed at the protein level, along with IL-6, IL-10, and TNF, suggesting an intensely inflammatory environment. Expression of caspase- 1 mRNA coupled with robust IL- $1 \beta$ production indicates inflammasome activation in TAs. IL-1 $\beta$ can be present early after birth in the setting of chorioamnionitis (35) and may contribute to high CXCL8 concentrations via its action on airway epithelial cells (5). Abundant expression of CXCL8, a neutrophil chemoattractant, and CCL2, important to monocyte, 

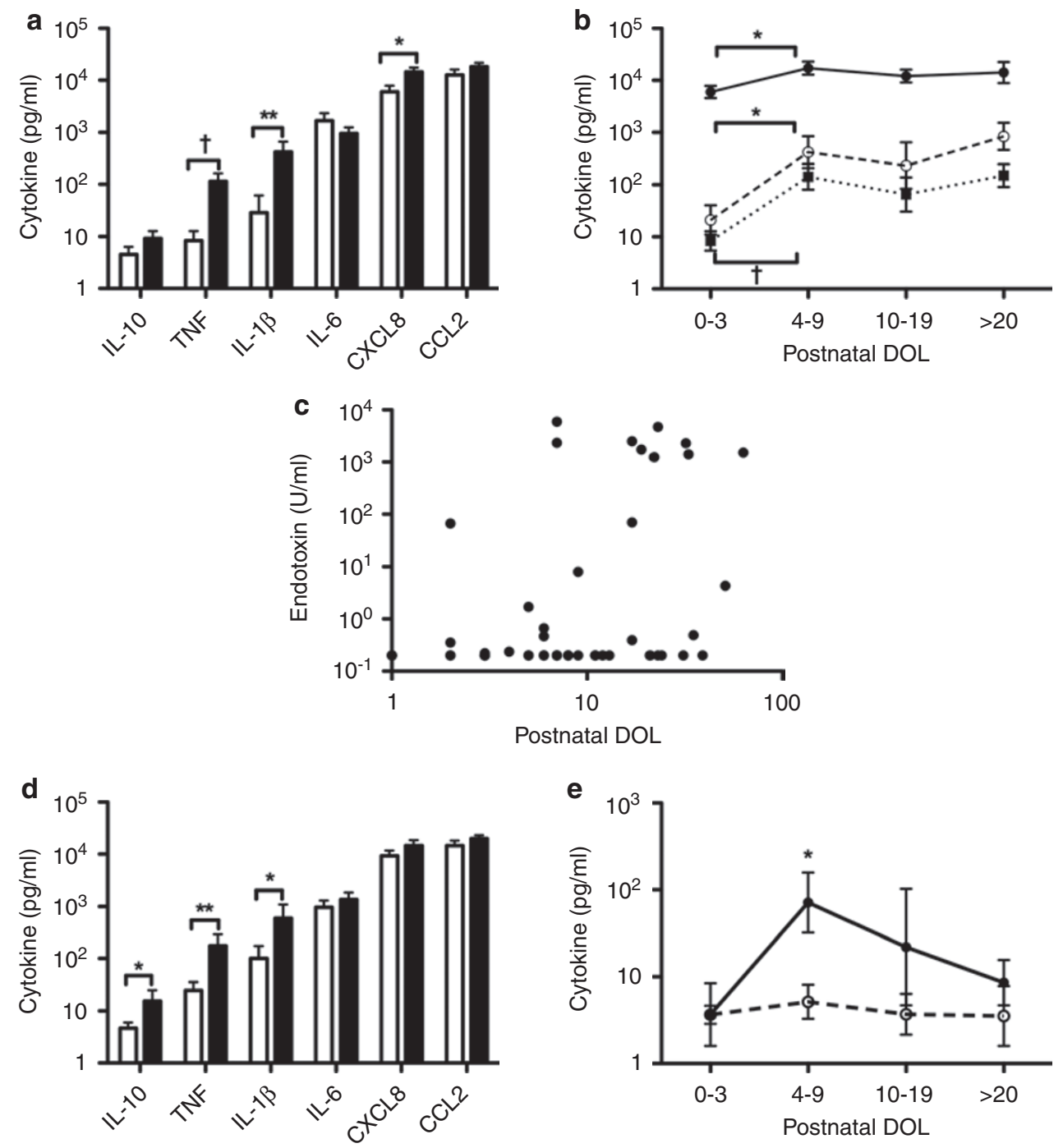

Figure 5. Endotoxin in TA supernatants correlates with postnatal DOL and cytokine concentrations. (a) TA supernatants from neonates with postnatal DOL $\geq 4 \mathrm{~d}(n=12$, mean $\mathrm{GA}=32.5 \pm 5.3 \mathrm{wk}$, solid bars) demonstrated higher levels of TNF, IL-1 $\beta$, and CXCL8 than neonates with postnatal DOL $<4 \mathrm{~d}(n=27-29$, mean GA $=26.3 \pm 2.1$ wk, open bars). (b) TNF, IL-1 $\beta$, and CXCL8 protein levels shown in relation to DOL 0-3 $(n=12), 4-9(n=12-15), 10-19(n=10)$, and $>20(n=9)$. TNF (square with dotted line), IL-1 $\beta$ (open circle with dashed line), and CXCL8 (solid circle with solid line) increased with DOL 0-3 and remained elevated through DOL $>20$. (c) The presence of detectable endotoxin was positively correlated with postnatal DOL $(n=53 ;$ Spearman $r=0.37, P<0.01)$.

(d) Endotoxin-positive TA supernatants ( $n=18$ to 19 , mean GA $=25.8 \pm 1.9 \mathrm{wk}$, solid bars) contained greater IL-10, TNF, and IL- $1 \beta$ concentrations than endotoxin-negative supernatants ( $n=24-27$, mean GA $29.5 \pm 4.7 \mathrm{wk}$, open bars). (e) IL-10 levels increased significantly in endotoxin-positive ( $n=2-7$, solid circles) TAs from DOL 0-3 to DOL 4-9 compared to endotoxin-negative ( $n=12-15$, open circles) TAs. Mann-Whitney unpaired $t$ test applied for all comparisons, " $P<0.05$, ${ }^{*} P<0.01$, and ${ }^{+} P<0.001$. DOL, day of life, GA, gestational age; IL, interleukin; LAL, Limulus amoebocyte lysate; TA, tracheal aspirate; TNF, tumor necrosis factor.

macrophage, and T-cell infiltration, is consistent with other studies $(4,36)$.

Several lines of evidence suggest that endotoxin is bioactive in TAs and could contribute to respiratory inflammation in our study cohort: (i) endotoxin is detected by the Limulus amoebocyte lysate assay and correlates with positive culture for Gram-negative microorganisms; (ii) mRNA transcripts encoding MD2, CD14, and TLR4 (components of the endotoxin receptor), as well as downstream MyD88 and NFkB, were abundantly expressed, indicating the presence of key endotoxin-detecting machinery; (iii) endotoxin-inducible antimicrobial proteins and peptides such as S100A12 were robustly expressed; (iv) multiple endotoxin-inducible cytokines were present; (v) endotoxin-positive TAs had higher transcriptional activation of several inflammatory cytokines, including TNF; and (vi) endotoxin-positive TAs demonstrated higher protein concentrations of TNF and IL-1 $\beta$. Greater postnatal age was positively correlated with both endotoxin positivity and increased cytokine levels, suggesting a possible role for length of intubation (37) and an increased risk of endotoxin-driven inflammation. Alternatively, as the group with lower postnatal age was also more premature (mean GA 26.3 vs. $32.5 \mathrm{wk}$ ), increased cytokine concentrations with advancing postnatal age in our study population could also reflect the ontogeny/ maturation of the inflammatory response in this age group. Thus, although cause and effect with regard to length of 
intubation (postnatal age in this patient group), prematurity (GA), and endotoxin positivity cannot be clearly determined, it is nonetheless evident that all these factors can contribute to cytokine elevation. Overall, these observations support the hypothesis that prolonged mechanical ventilation contributes to endotoxin exposure and inflammatory sequelae $(1,3)$.

In sum, we have developed a novel qRT-PCR transcriptional approach to characterize broad innate immune activation in neonatal TAs, defined relative magnitude of expression within classes of innate immune components, identified S100A12 as a novel TA biomarker of PMN-driven inflammation, and provided evidence that bioactive endotoxin in TAs may contribute to inflammation, including activation of the NFKB signaling cascade that has been linked to bronchopulmonary dysplasia (38). In light of the likely contribution of bioactive endotoxin to innate immune activation, inflammation, and respiratory disease in this setting, endotoxin antagonists (26) - several of which are under biopharmaceutical development (39), including human clinical trials, and can be administered via the respiratory route (40-42) - may be beneficial to reduce pulmonary inflammation in critically ill preterm newborns.

\section{METHODS}

\section{Collection and Processing of Neonatal TAs}

TAs were collected from mechanically ventilated newborn infants (regardless of GA or birth weight) who were intubated for respiratory failure at the Brigham and Women's Hospital (BWH) and the Beth Israel Deaconess Medical Center (BIDMC) newborn intensive care units (NICUs) as previously described (24), and in accordance with BWH and BIDMC IRB-approved protocols. Briefly, TA samples were collected from mechanically ventilated newborns BWH and BIDMC NICUs. Informed consent was not required as TAs are discarded specimens. TAs were collected after endotracheal instillation of $0.5 \mathrm{ml} / \mathrm{kg}$ sterile, pyrogen-free saline using sterile suction catheters and placed on ice for processing within $4 \mathrm{~h}$. Because of TA volume constraints, subsets of samples were analyzed for different markers, with number $(n)$ provided in each figure legend. Where available, mean levels of $\mathrm{FiO}_{2}$ exposure over total intubation period and TA bacterial culture results were obtained and compiled (bacterial cultures $\pm 7 \mathrm{~d}$ of collection of study samples, data not shown).

\section{Purification of TA Pellet RNA}

All pipettes/surfaces were cleaned with RNase Zap (Applied Biosystems/Ambion, Austin, TX) to prevent degradation of RNA. TAs were transferred to a $1.5-\mathrm{ml}$ polypropylene collection tube and centrifuged $(700 \mathrm{~g}$, for $5 \mathrm{~min}$ at room temperature). TA supernatant aliquots were stored at $-80^{\circ} \mathrm{C}$. Cells were counted and viability assessed $(\geq 85 \%)$ by trypan blue exclusion. TA cell pellet RNA was preserved by adding $375 \mu$ l RNA Cell Protect Reagent (QIAGEN, Valencia, CA). TA cell pellet RNA was processed immediately or stored at $-80^{\circ} \mathrm{C}$. RNA was isolated by RNA Protect Cell Mini and RNeasy MinElute Cleanup Kits (QIAGEN) per the manufacturer's instructions. TA mRNA yield positively correlated with TA cellularity as measured by hemocytometry (data not shown). RNA concentration was measured using a spectrophotometer (absorbance 260/280) or Nanodrop 1000 spectrophotometer (Thermo Fisher Scientific, Waltham, MA). RNA purity was assessed by Nanodrop with A260/280 ratios ranging from 1.8-2.0 and A260/230 ratios from 2.0-2.2 deemed pure according to the manufacturer's guidelines (http://www.nanodrop.com).

\section{Quantitative Real-Time PCR}

Total mRNA (100 ng) was reverse-transcribed to cDNA using the $\mathrm{RT}^{2}$ First Strand Kit (QIAGEN) according to the manufacturer's instructions. The equivalent of $\sim 1 \mathrm{ng}$ RNA/well was assayed using the $\mathrm{RT}^{2}$ Profiler PCR Array System (QIAGEN) according to the manufacturer's instructions. A modified Human Innate and Adaptive Immune Response $\mathrm{RT}^{2}$ Profiler PCR array was created (CAPH09693-PAHS-052; QIAGEN) containing primers for 86 genes of interest (including those for bactericidal/permeability increasing protein and CXCL8) and 10 controls (5 housekeeping genes, including those for $\beta$-2-microglobulin, hypoxanthine phosphoribosyl transferase 1, ribosomal protein L13a, GAPDH, and ACTB, as well as human genomic DNA contamination-, reverse transcription-, and positive PCR-controls). mRNA levels were normalized to housekeeping genes and quantified using the $\Delta \Delta$ comparative threshold (Ct) method (43), using an analysis tool from QIAGEN (http://www.sabiosciences.com/pcr/arrayanalysis.php). To compare mRNA abundance, transcripts were expressed as a ratio of the $2^{-\Delta \mathrm{Ct}}$ value to the $2^{-\Delta \mathrm{Ct}}$ of the least abundant transcript (lipopolysaccharide binding protein, average $\mathrm{Ct}=38.976$ ). Statistical analyses comparing mRNA levels of different transcripts were applied when mean transcript levels differed by $>5.5$ cycles (i.e., $>44$-fold difference), as advised by the associate director of Bioinformatics, Biological Content Development, R\&D Americas, QIAGEN.

\section{Milliplex Cytokine Assays}

TA protein levels of IL-1 $\beta$, IL-6, CXCL8, IL-10, CCL2, and TNF were measured using a customized Milliplex Human Cytokine Immunoassay Kit (Millipore, Chicago, IL). using a Milliplex Analyzer 3.1 Luminex 200 machine (Millipore) and analyzed on corresponding software according to the manufacturer's instructions.

\section{Endotoxin Measurement}

Endotoxin was measured by Limulus amoebocyte lysate (Charles River, Boston, MA) assay according to the manufacturer's instructions.

\section{Total Protein Measurement}

Total protein was measured using the Bradford protein assay (BioRad Laboratories, Hercules, CA) with human serum albumin as standard (Talecris Biotherapeutics, Research Triangle Park, NC).

\section{Measurement of S100A12}

S100A12 in TA supernatants was measured by enzyme-linked immunosorbent assay according to the manufacturer's instructions (CycLex, Nagano, Japan).

\section{Flow Cytometry}

After TA cell collection, Fc binding sites were blocked using 10\% normal human serum (60 min, room temperature). PMNs were stained (20 min, room temperature) with FITC-conjugated anti-CD66b $\mathrm{mAb}$ (mouse IgM, clone G10F5; BD Pharmingen, San Diego, CA) at $10 \mu \mathrm{l} / 5 \times 10^{5}$ cells, or with FITC-conjugated isotype control $\mathrm{mAb}$ (mouse IgM, clone G155-228; BD Pharmingen). After washing (Stain Buffer; BD Pharmingen) and permeabilization $(1 \times$ fluorescence activated cell sorter lysing solution or $1 \times$ permeabilization/wash buffer containing saponin; BD Pharmingen, $20 \mathrm{~min}$, room temperature), S100A12 was detected with mouse IgG1 $\mathrm{\kappa}$ anti-S100A12 mAb (clone 19F5; Santa Cruz Biotechnology, CA; $1 \mu \mathrm{l} / 5 \times 10^{5}$ cells) or mouse $\operatorname{IgG1}(\kappa)$ isotype control $\mathrm{mAb}$ (clone MOPC-21; BD Pharmingen), followed by incubation ( $30 \mathrm{~min}$, room temperature) with a second-

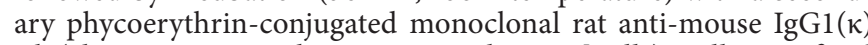
$\mathrm{Ab}$ (clone A85-I; BD Pharmingen; $5 \mu \mathrm{l} / 5 \times 10^{5}$ cells). Cells were fixed ( $1 \%$ formalin and stain buffer, $4^{\circ} \mathrm{C}$ ) before analysis by flow cytometry (Cytomation MoFlo flow cytometer, DAKO, Carpinteria, CA) and analyzed with FloJo Flow Cytometry Analysis Software (Tree Star, Ashland, OR).

\section{Fluorescent/Confocal Microscopy}

Formalin-fixed TA cells were stained with DRAQ5 (Alexis Biochemicals, Farmingdale, NY) nuclear stain 10 min before imaging on a Nikon TE-2000 inverted microscope fitted with a video-rate confocal system (Yokogawa, Sugar Land, TX) paired with a $\mathrm{Kr} / \mathrm{Ar}$ laser (Melles Griot, Albuquerque, NM), fluorescence filters, and an Orca camera (Hamamatsu, Bridgewater, NJ) for image digitization. Slidebook Software (Intelligent Imaging Innovations, Denver, CO) was used for image capture and 3-dimensional rendering. Confocal images were collected either as single 2-dimensional planes or 
3 -dimensional stacks using a $100 \times$ oil immersion objective with an aperture of 1.4 and a focal step size of $0.3 \mu$.

\section{Statistics}

Statistical analyses of mRNA expression employed SA Biosciences software (QIAGEN) using a Student's $t$ test of the replicate $2^{-\Delta C t}$ values for each gene in the endotoxin-negative and endotoxin-positive sample sets (Figure 4a). Graphpad Prism 5.0a Software (GraphPad Software, La Jolla, CA) was used to perform statistical analysis for TA supernatant biomarkers and relative mRNA abundance. Data groups with unequal variances were analyzed by Mann-Whitney unpaired $t$ test. For multiple comparisons, one-way ANOVA repeated-measures test with Bonferroni's multiple-comparison posttest correction was used. Correlations were drawn using the Spearman method. $P$ values $<0.05$ were considered significant.

\section{ACKNOWLEDGMENTS}

We thank the nurses and respiratory therapists of the Brigham and Women's Hospital and Beth Israel Deaconess Medical Center neonatal intensive care units for assistance with sample collection and Leslie Kalish of the Children's Hospital Boston Clinical Research Program for statistical advice. We also thank Jessica Wagner of the Imaging Core at the Harvard Digestive Diseases Center.

\section{STATEMENT OF FINANCIAL SUPPORT}

This study was supported by a National Institutes of Health (NIH) Specialized Center of Research (SCOR) grant (HL72931), an NIH National Center for Research Resources K30 grant (RR022292-07), an NIH RO1 Grant Al067353-01A1 (to O.L.), and a Thrasher Research Fund Early Career Award (to K.E.N.).

\section{REFERENCES}

1. Jobe AH. Antenatal associations with lung maturation and infection. J Perinatol 2005;25:Suppl 2:S31-5.

2. Starner TD, Agerberth B, Gudmundsson GH, McCray PB Jr. Expression and activity of beta-defensins and LL-37 in the developing human lung. J Immunol 2005;174:1608-15.

3. De Dooy J, Ieven M, Stevens W, Schuerwegh A, Mahieu L. Endotracheal colonization at birth is associated with a pathogen-dependent pro- and antiinflammatory cytokine response in ventilated preterm infants: a prospective cohort study. Pediatr Res 2004;56:547-52.

4. De Dooy J, Ieven M, Stevens W, De Clerck L, Mahieu L. High levels of CXCL8 in tracheal aspirate samples taken at birth are associated with adverse respiratory outcome only in preterm infants younger than 28 weeks gestation. Pediatr Pulmonol 2007;42:193-203.

5. Shimotake TK, Izhar FM, Rumilla K, et al. Interleukin (IL)- 1 beta in tracheal aspirates from premature infants induces airway epithelial cell IL-8 expression via an NF-kappa B dependent pathway. Pediatr Res 2004;56:907-13.

6. Wynn J, Cornell TT, Wong HR, Shanley TP, Wheeler DS. The host response to sepsis and developmental impact. Pediatrics 2010;125:1031-41.

7. Levy O. Innate immunity of the newborn: basic mechanisms and clinical correlates. Nat Rev Immunol 2007;7:379-90.

8. O'Neill LA. The interleukin-1 receptor/Toll-like receptor superfamily: 10 years of progress. Immunol Rev 2008;226:10-8.

9. Ogura Y, Sutterwala FS, Flavell RA. The inflammasome: first line of the immune response to cell stress. Cell 2006;126:659-62.

10. Wesche H, Henzel WJ, Shillinglaw W, Li S, Cao Z. MyD88: an adapter that recruits IRAK to the IL-1 receptor complex. Immunity 1997;7: 837-47.

11. Palmer CD, Mutch BE, Workman S, McDaid JP, Horwood NJ, Foxwell BM. Bmx tyrosine kinase regulates TLR4-induced IL-6 production in human macrophages independently of p38 MAPK and NFkapp $\}$ B activity. Blood 2008;111:1781-8.

12. Severa M, Fitzgerald KA. TLR-mediated activation of type I IFN during antiviral immune responses: fighting the battle to win the war. Curr Top Microbiol Immunol 2007;316:167-92.

13. Poltorak A, Smirnova I, He X, et al. Genetic and physical mapping of the Lps locus: identification of the toll-4 receptor as a candidate gene in the critical region. Blood Cells Mol Dis 1998;24:340-55.
14. Shimazu R, Akashi S, Ogata H, et al. MD-2, a molecule that confers lipopolysaccharide responsiveness on Toll-like receptor 4. J Exp Med 1999;189:1777-82.

15. Williams LM, Ricchetti G, Sarma U, Smallie T, Foxwell BM. Interleukin-10 suppression of myeloid cell activation-a continuing puzzle. Immunology 2004;113:281-92.

16. Deshmane SL, Kremlev S, Amini S, Sawaya BE. Monocyte chemoattractant protein-1 (MCP-1): an overview. J Interferon Cytokine Res 2009;29:313-26.

17. Kobayashi Y. Neutrophil infiltration and chemokines. Crit Rev Immunol 2006;26:307-16.

18. McDonald JR, Finck BK, McIntosh LM, Wilson SE. Anti-inflammatory approaches that target the chemokine network. Recent Pat Inflamm Allergy Drug Discov 2011;5:1-16.

19. Destin KG, Linden JR, Laforce-Nesbitt SS, Bliss JM. Oxidative burst and phagocytosis of neonatal neutrophils confronting Candida albicans and Candida parapsilosis. Early Hum Dev 2009;85:531-5.

20. Ganz T. Antimicrobial polypeptides in host defense of the respiratory tract. J Clin Invest 2002;109:693-7.

21. Neff SB, Z'graggen BR, Neff TA, et al. Inflammatory response of tracheobronchial epithelial cells to endotoxin. Am J Physiol Lung Cell Mol Physiol 2006;290:L86-96.

22. Ricchetti GA, Williams LM, Foxwell BM. Heme oxygenase 1 expression induced by IL-10 requires STAT- 3 and phosphoinositol-3 kinase and is inhibited by lipopolysaccharide. J Leukoc Biol 2004;76:719-26.

23. Ye S, Goldsmith EJ. Serpins and other covalent protease inhibitors. Curr Opin Struct Biol 2001;11:740-5.

24. Nathe KE, Parad R, Van Marter LJ, et al. Endotoxin-directed innate immunity in tracheal aspirates of mechanically ventilated human neonates. Pediatr Res 2009;66:191-6.

25. Bouchon A, Facchetti F, Weigand MA, Colonna M. TREM-1 amplifies inflammation and is a crucial mediator of septic shock. Nature 2001;410:1103-7.

26. Palmer CD, Mancuso CJ, Weiss JP, Serhan CN, Guinan EC, Levy O. $17^{\circ}$ Resolvin D1 differentially regulates TLR4-mediated responses of primary human macrophages to purified LPS and live E. coli. J Leukoc Biol 2011;90:459-70.

27. Vogl T, Pröpper C, Hartmann M, et al. S100A12 is expressed exclusively by granulocytes and acts independently from MRP8 and MRP14. J Biol Chem 1999;274:25291-6.

28. Guignard F, Mauel J, Markert M. Identification and characterization of a novel human neutrophil protein related to the S100 family. Biochem J 1995;309 (Pt 2):395-401.

29. Wittkowski H, Sturrock A, van Zoelen MA, et al. Neutrophil-derived S100A12 in acute lung injury and respiratory distress syndrome. Crit Care Med 2007;35:1369-75.

30. Talwar S, Munson PJ, Barb J, et al. Gene expression profiles of peripheral blood leukocytes after endotoxin challenge in humans. Physiol Genomics 2006;25:203-15.

31. Cole AM, Kim YH, Tahk S, et al. Calcitermin, a novel antimicrobial peptide isolated from human airway secretions. FEBS Lett 2001;504:5-10.

32. Foell D, Wittkowski H, Vogl T, Roth J. S100 proteins expressed in phagocytes: a novel group of damage-associated molecular pattern molecules. J Leukoc Biol 2007;81:28-37.

33. Pietzsch J, Hoppmann S. Human S100A12: a novel key player in inflammation? Amino Acids 2009;36:381-9.

34. Lorenz E, Muhlebach MS, Tessier PA, et al. Different expression ratio of S100A8/A9 and S100A12 in acute and chronic lung diseases. Respir Med 2008;102:567-73.

35. Cayabyab RG, Jones CA, Kwong KY, et al. Interleukin-1beta in the bronchoalveolar lavage fluid of premature neonates: a marker for maternal chorioamnionitis and predictor of adverse neonatal outcome. J Matern Fetal Neonatal Med 2003;14:205-11.

36. Baier RJ, Majid A, Parupia H, Loggins J, Kruger TE. CC chemokine concentrations increase in respiratory distress syndrome and correlate with development of bronchopulmonary dysplasia. Pediatr Pulmonol 2004;37:137-48. 
37. Jónsson B, Tullus K, Brauner A, Lu Y, Noack G. Early increase of TNF alpha and IL-6 in tracheobronchial aspirate fluid indicator of subsequent chronic lung disease in preterm infants. Arch Dis Child Fetal Neonatal Ed 1997;77:F198-201.

38. Benjamin JT, Carver BJ, Plosa EJ, et al. NF-kappaB activation limits airway branching through inhibition of Sp1-mediated fibroblast growth factor-10 expression. J Immunol 2010;185:4896-903.

39. Guinan EC, Barbon CM, Kalish LA, et al. Bactericidal/permeability-increasing protein (rBPI21) and fluoroquinolone mitigate radiation-induced bone marrow aplasia and death. Sci Transl Med 2011;3:110ra118.

40. Palmer CD, Guinan EC, Levy O. Deficient expression of bactericidal/permeability-increasing protein in immunocompromised hosts: translational potential of replacement therapy. Biochem Soc Trans 2011;39:994-9.
41. Tidswell M, LaRosa SP. Toll-like receptor-4 antagonist eritoran tetrasodium for severe sepsis. Expert Rev Anti Infect Ther 2011;9:507-20.

42. Srivastava A, Casey H, Johnson N, Levy O, Malley R. Recombinant bactericidal/permeability-increasing protein $\mathrm{rBPI} 21$ protects against pneumococcal disease. Infect Immun 2007;75:342-9.

43. Bustin SA. Absolute quantification of mRNA using real-time reverse transcription polymerase chain reaction assays. J Mol Endocrinol 2000;25:169-93.

(ब) This work is licensed under the Creative Derivative Works 3.0 Unported License. To view a copy of this license, visit http://creativecommons.org/ licenses/by-nc-nd/3.0/ 